\title{
ON THE FACTORIZATIONS OF ORDINARY LINEAR DIFFERENTIAL OPERATORS
}

\author{
G. J. ETGEN, G. D. JONES AND W. E. TAYLOR, JR.
}

\begin{abstract}
Relations are found between the nonvanishing of certain Wronskians and disconjugacy properties of $L_{n} y+p y=0$, where $L_{n} y$ is a disconjugate operator and $p$ is sign definite. The results are then used to show ways in which $L_{n} y+p y$ can be factored.
\end{abstract}

Introduction. In this paper we investigate possible ways of factoring certain $n$th order linear differential operators of the form

$$
L y=P_{n} y^{(n)}+P_{n-1} y^{(n-1)}+\cdots+P_{1} y^{\prime}+P_{0} y,
$$

where $P_{i}(x)$ are real valued and $P_{n}(x) \neq 0$, into products of lower order operators of the same type, where the lower order operators are irreducible.

According to a well-known result of Pólya [6], the operator (1) can be factored into a product of first order operators on an interval $I$ if and only if it is disconjugate there. Consequently, we will investigate (1) when it is not disconjugate.

Other work on the factoring of (1) can be found in $[\mathbf{1}, \mathbf{7}, \mathbf{8}$, and 9]. We will utilize the following result on factoring due to Zettl [8].

THEOREM 1. Suppose $1 \leq k<n$. The operator (1) has a factorization $L=R Q$ where $R$ and $Q$ are of the same type with $Q$ of order $k$ and $R$ of order $n-k$ on an interval $I$ if and only if there are $k$ solutions $y_{1}, \ldots, y_{k}$ of $L y=0$ which satisfy $\omega\left(y_{1}, \ldots, y_{k}\right) \neq 0$ on $I$, where $\omega\left(y_{1}, \ldots, y_{k}\right)$ is the Wronskian of $y_{1}, \ldots, y_{k}$. Further, $Q$ has the representation

$$
Q y=\omega\left(y_{1}, \ldots, y_{k}, y\right) \text {. }
$$

1. In this section we will list some of the main definitions and results that will be used later for the equations $L y=0$, where

$$
L y=L_{n} y+p(x) y .
$$

Throughout, we will assume

$$
p(x) \text { is continuous, real valued and strictly of one sign, and } L_{n} \text { is }
$$
the disconjugate $n$th order differential operator

$$
L_{n} y=\rho_{n}\left(\rho_{n-1}, \ldots,\left(\rho_{1}\left(\rho_{0} y\right)^{\prime} \cdots\right)^{\prime}\right.
$$

with $\rho_{i}>0$ and $\rho_{i} \in C^{n-i}$. We let $L_{0} y=\rho_{0} y, L_{i} y=\rho_{i}\left(L_{i-1} y\right)^{\prime}, i=1, \ldots, n$, and call $L_{0} y, \ldots, L_{n} y$ quasi-derivatives of $y$.

Received by the editors September 30, 1985.

1980 Mathematics Subject Classification. Primary 34C10; Secondary 34B05.

Key words and phrases. Linear differential equation, oscillation, disconjugate, factorization, linear differential operator. 
DEFINITION 1 (ELIAS $[\mathbf{2}]$ ). Let $\sigma\left(C_{0}, \ldots, C_{n}\right)$ denote the number of sign changes in the sequence $C_{0}, \ldots, C_{n}$ of nonzero numbers. Then for a solution $y \not \equiv 0$ of

$$
L_{n} y+p(x) y=0
$$

and a point $x$ we define

$$
S\left(y, x^{+}\right)=\lim _{t \rightarrow x^{+}} \sigma\left(L_{0} y(t),-L_{i} y(t), \ldots,(-1)^{n} L_{n} y(t)\right)
$$

and

$$
S\left(y, x^{-}\right)=\lim _{t \rightarrow x^{-}} \sigma\left(L_{0} y(t), L_{1} y(t), \ldots, L_{n} y(t)\right) .
$$

Let $a \leq x_{1} \leq \cdots \leq x_{r} \leq b$ be the zeros of the quasi-derivatives $L_{0} y, L_{1} y, \ldots$, $L_{n-1} y$ of a solution $y$ of $(4)$ in $[a, b]$, where the same $x_{i}=c$ is used to denote zeros of two different quasi-derivatives $L_{j} y$ and $L_{k} y$ if and only if $L_{j} y(c)=L_{k} y(c)$ implies either $L_{l} y(c)=0$ for all $j \leq l \leq k$ or $L_{l} y(c)=0$ for all $k \leq l \leq n-1$ and $0 \leq l \leq j$. With $n\left(x_{i}\right)$ denoting the number of consecutive (with $L_{0} y$ following $\left.L_{n-1} y\right)$ quasi-derivatives which vanish at $x_{i}$, and $\langle q\rangle$ denoting the greatest even integer not greater than $q$, we state the following theorem due to Elias [2].

THEOREM 2. Every solution y of (4) satisfies the condition

$$
N(y) \equiv S\left(y, a^{+}\right)+S\left(y, b^{-}\right)+\sum_{a<x_{t}<b}\left\langle n\left(x_{t}\right)\right\rangle \leq n .
$$

Moreover, $S\left(y, b^{-}\right)$and $n-S\left(y, a^{+}\right)$are both even if $p(x)<0$ and both odd if $p(x)>0$. If $N(y)=n$, then $L_{t+1} y$ has exactly one sign change between two consecutive zeros of $L_{t} y$ in $[a, b]$. In addition, $L_{t+1} y$ changes sign before the first zero of $L_{t} y$ in $(a, b]$ if and only if $\operatorname{sgn}\left[L_{t+1} y(a+\varepsilon)\right]=\operatorname{sgn}\left[L_{t} y(a+\varepsilon)\right]$, and this sign change is unique. The situation is similar near the endpoint $b$.

DEFINITION 2. Equation (4) is $(k, n-k)$ disconjugate on $[a, b]$ provided there is no solution $y$ with zeros of order at least $k$ and $n-k$ at $a$ and $b$, respectively. Equation $(4)$ is $(k, n-k)$ disconjugate on $[a,+\infty)$ if it is $(k, n-k)$ disconjugate on $[a, b]$ for all $a<b<\infty$.

In the discussion that follows, we will let $\gamma<n$ be an integer such that $n-\gamma$ is odd for $p(x)<0$ and even for $p(x)>0$. Hence by Theorem 2, (4) will be $(\gamma, n-\gamma)$ disconjugate.

We will give our results using quasi-derivatives rather than derivatives of $y$. Thus

$$
W\left(y_{1}, y_{2}, \ldots, y_{r}\right)(x)=\left|\begin{array}{ccc}
L_{0} y_{1}(x) & \cdots & L_{0} y_{r}(x) \\
L_{1} y_{1}(x) & \cdots & L_{1} y_{r}(x) \\
\vdots & & \vdots \\
L_{r-1} y_{1}(x) & \cdots & L_{r-1} y_{r}(x)
\end{array}\right|
$$

We will say $y$ has a zero of order $r$ at $a$ if $L_{0} y(a)=\cdots L_{1} y(a)=\cdots=L_{r-1} y(a)=$ 0 . Theorem 1 holds and will be used with $W$ replacing $\omega$.

2. In this section we prove the existence of a basis for the solution space of (4) with certain properties that will be used later. 
THEOREM 3. There are linearly independent solutions $y_{i}$ of $(4)$ for $i=0,1, \ldots$, $n-1$ with the following properties:

1. $y_{i}$ has a zero of multiplicity exactly $i$ at $x=a$.

2. If $b<\infty$, then $y_{i}$ has a zero of multiplicity at least $n-1-\left[i+\left(1+(-1)^{i-\gamma}\right) / 2\right]$ at $x=b$.

3. $z \in \operatorname{span}\left[y_{\gamma+2 r}, \ldots, y_{\gamma+2 s+1}\right]$ for $0 \leq r \leq s<(n-\gamma-1) / 2$ implies $\gamma+2 r+1 \leq$ $S\left(z, x^{+}\right) \leq \gamma+2 s+1$ and $n-(\gamma+2 s+1) \leq S\left(z, x^{-}\right) \leq n-(\gamma+2 r+1)$ for $x \in(a, b)$, where $b \leq \infty$.

4. $W\left(y_{\gamma+2 r}, \ldots, y_{\gamma+2 s+1}\right)(x) \neq 0$ for $x \in(a, b)$.

PROOF. At first we will assume $b<\infty$. In that case, let $y_{\gamma+2 j}$ be the nontrivial solution of (4) satisfying

$$
\begin{array}{ll}
L_{i} y_{\gamma+2 j}(a)=0 & \text { for } i=0, \ldots, \gamma+2 j-1, \gamma+2 j+1, \\
L_{i} y_{\gamma+2 j}(b)=0 & \text { for } i=0, \ldots, n-\gamma-2 j-3 .
\end{array}
$$

Let $y_{\gamma+2 j+1}$ be the nontrivial solution of (4) satisfying

$$
\begin{array}{ll}
L_{i} y_{\gamma+2 j+1}(a)=0 & \text { for } i=0, \ldots, 2 j+\gamma \\
L_{i} y_{\gamma+2 j+1}(b)=0 & \text { for } i=0, \ldots, n-\gamma-2 j-3 .
\end{array}
$$

In any case define $y_{0}$ and $y_{n-1}$ as in (i) or (ii), depending on whether $n-\gamma$ is even or odd. Clearly $y_{i}$ has a zero of multiplicity $i$ at $a$ and satisfies condition (2). If $y_{i}$ has a zero of multiplicity greater than $i$ at $a$ for $i=\gamma+2 j$ or $i=\gamma+2 j+1$, then according to Theorem 2

$$
n \geq S\left(y_{i}, a^{+}\right)+S\left(y_{i}, b^{-}\right) \geq(\gamma+2 j+3)+(n-\gamma-2 j-2)=n+1 .
$$

Thus the $y_{i}$ satisfy conditions (1), (2) and are linearly independent.

Let $z \in \operatorname{span}\left[y_{\gamma+2 r}, \ldots, y_{\gamma+2 s+1}\right]$. Since $S\left(z, x^{+}\right)$is a nondecreasing function of $x$ which assumes values of the form $\gamma+2 j+1$ for $j$ an integer and since $z$ has a zero at $a$ of multiplicity at least $\gamma+2 r$, it follows that $\gamma+2 r+1 \leq S\left(z, x^{+}\right)$for $x \geq a$. Since $S\left(z, x^{-}\right)$is nonincreasing and $z$ has a zero at $b$ of multiplicity at least $n-2 s-\gamma-1$, it follows that $S\left(z, x^{-}\right) \geq n-2 s-\gamma-1$ for $x \leq b$. If $\bar{x} \in(a, b)$ is such that $L_{i} z(\bar{x}) \neq 0$ for $i=0, \ldots, n-1$, then

$$
\gamma+2 r+1 \leq S\left(z, \bar{x}^{+}\right)=n-S\left(z, \bar{x}^{-}\right) \leq n-[n-2 s-\gamma-1]=2 s+\gamma+1 .
$$

Also

$$
n-2 s-\gamma-1 \leq S\left(z, \bar{x}^{-}\right)=n-S\left(z, \bar{x}^{+}\right) \leq n-(\gamma+2 r+1)
$$

Since such points are dense in $(a, b)$, property (3) follows.

Suppose there is an $x^{*} \in(a, b)$ such that $W\left(y_{\gamma+2 r}, \ldots, y_{\gamma+2 s+1}\right)\left(x^{*}\right)=0$. Then there is a nontrivial $z \in \operatorname{span}\left(y_{\gamma+2 r}, \ldots, y_{\gamma+2 s+1}\right)$ with a zero of multiplicity $2 s-$ $2 r+2$ at $x^{*}$. Thus

$n \geq S\left(z, a^{+}\right)+\left\langle n\left(x^{*}\right)\right\rangle+S\left(z, b^{-}\right) \geq(\gamma+2 r+1)+(2 s-2 r+2)+(n-2 s-\gamma-1)=n+2$.

Thus property (4) follows.

To prove the theorem for the case $b=+\infty$, we first apply the above arguments to the functions $y_{i}(x, n)$ on the interval $[a, n]$. Then using standard compactness arguments, we let $y_{i}(x)=\lim _{j \rightarrow \infty} y_{i}\left(x, m_{j}\right)$ where $m_{j} \rightarrow \infty$. Conditions (1), (3) and (4) are easily shown to hold for the functions $y_{i}$. 
3. If $(4)$ is $(\gamma+1, n-\gamma-1)$ disconjugate on $[a, b]([a,+\infty))$, then according to [2] $y_{\gamma+1}$ (given in Theorem 3 ) has no zeros in $(a, b)((a,+\infty))$. In this section we extend that result to show that the odd order Wronskian of $y_{\gamma+1}, y_{\gamma+2}, \ldots, y_{\gamma+2 k+1}$ for $0 \leq k \leq(n-\gamma-1) / 2$ has no zeros in $(a, b)((a,+\infty))$.

We will let $\left\{y_{i}: 0 \leq i \leq n-1\right\}$ be solutions of (4) satisfying the conditions of Theorem 3. For $s \in(a, b)$ we define

$$
U(x, t)=\left|\begin{array}{ccc}
L_{0} y_{\gamma}(s) & \cdots & L_{0} y_{\gamma+2 k+1}(s) \\
\vdots & & \vdots \\
L_{2 k} y_{\gamma}(s) & & L_{2 k} y_{\gamma+2 k+1}(s) \\
L_{\gamma} y_{\gamma}(x) & & L_{\gamma} y_{\gamma+2 k+1}(x)
\end{array}\right| .
$$

THEOREM 4. Suppose for some $s_{0} \in(a, b), U\left(a, s_{0}\right)=0$. Then the function $x(s)$ defined by $U(x, s)=0$, where $x\left(s_{0}\right)=a$, is such that $d x /\left.d s\right|_{s=s_{0}}>0$.

PROOF. Define a solution $u_{2}(x, s)$ of $(4)$ in $x$ by

$$
u_{2}(x, s) \equiv\left|\begin{array}{ccc}
L_{0} y_{\gamma}(s) & \cdots & L_{0} y_{\gamma+2 k+1}(s) \\
\vdots & & \vdots \\
L_{2 k} y_{\gamma}(s) & & L_{2 k} y_{\gamma+2 k+1}(s) \\
y_{\gamma}(x) & & y_{\gamma+2 k+1}(x)
\end{array}\right| .
$$

Suppose

$$
L_{\gamma+1} u_{2}\left(a, s_{0}\right)=0 .
$$

Then there is a solution $v_{1}$ of $(4)$ in $\operatorname{span}\left\{y_{\gamma}, \ldots, y_{\gamma+2 k+1}\right\}$ with zeros of multiplicities $\gamma, 2 k+1$, and $n-\gamma-2 k-2$ at $a, s_{0}$, and $b$, respectively. Moreover, $L_{\gamma+1} v_{1}(a)=$ 0 . Since $W\left(a, s_{0}\right)=0$, there is a solution $v_{2}$ of $(4)$ in $\operatorname{span}\left\{y_{\gamma}, \cdots, y_{\gamma+2 k+1}\right\}$ with zeros of multiplicities $\gamma+1,2 k+1$, and $n-\gamma-2 k-2$ at $a, s_{0}$, and $b$, respectively. If $v_{1}=v_{2}$, then $v_{2}$ has a zero of multiplicity $\gamma+2$ at $a$. Thus by Theorem 2

$$
n \geq S\left(v_{2}, a^{+}\right)+\left\langle n\left(s_{0}\right)\right\rangle+S\left(v_{2}, b^{-}\right) \geq(\gamma+3)+2 k+[n-(\gamma+2 k+1)]=n+2 .
$$

Thus $v_{1} \neq v_{2}$. But in that case there is a linear combination $v$ of $v_{1}$ and $v_{2}$ with a zero of multiplicity $2 k+2$ at $s_{0}$. Thus

$n \geq S\left(v, a^{+}\right)+\left\langle n\left(s_{0}\right)\right\rangle+S\left(v, b^{-}\right) \geq(\gamma+1)+(2 k+2)+[n-(\gamma+2 k+1)]=n+2$.

Hence $L_{\gamma+1} u_{2}\left(a, s_{0}\right) \neq 0$. Since $\partial U\left(a, s_{0}\right) / \partial x=L_{\gamma+1} U_{2}\left(a, s_{0}\right) / \rho_{\gamma+1}(a)$, it follows by the Implicit Function Theorem that $x(s)$ is uniquely defined and

$$
\frac{d x}{d s}=-\frac{\partial U}{\partial s} / \frac{\partial U}{\partial x}
$$

To show that $d x / d s$ is positive at $s=s_{0}$, we define

$$
\left.u_{1}(x, s) \equiv \mid \begin{array}{ccc}
L_{0} y_{\gamma}(s) & \cdots & L_{0} y_{\gamma+2 k+1}(s) \\
\vdots & & \vdots \\
L_{2 k-1} y_{\gamma}(s) & & L_{2 k-1} y_{\gamma+2 k+1}(s) \\
y_{\gamma}(x) & & y_{\gamma_{2} k+1(x)} \\
L_{\gamma} y_{\gamma}(a) & \cdots & L_{\gamma} y_{\gamma+2 k+1}(a)
\end{array}\right]
$$


and

$$
u_{3}(x, s) \equiv\left|\begin{array}{ccc}
L_{0} y_{\gamma}(s) & \cdots & L_{0} y_{\gamma+2 k+1}(s) \\
\vdots & & \vdots \\
L_{2 k-1} y_{\gamma}(s) & & L_{2 k-1} y_{\gamma+2 k+1}(s) \\
y_{\gamma}(x) & & y_{\gamma+2 k+1(x)} \\
L_{\gamma+1} y_{\gamma}(a) & \cdots & L_{\gamma+1} y_{\gamma+2 k+1}(a)
\end{array}\right|
$$

An application of (5) shows that any solution of (4) satisfying the boundary conditions of $u_{2}(x, s)$ is essentially unique. But since $U\left(a, s_{0}\right)=0, u_{1}\left(x, s_{0}\right)$ also satisfies those boundary conditions. Hence

$$
u_{1}\left(x, s_{0}\right)=k u_{2}\left(x, s_{0}\right)
$$

Applying (5) to $u_{3}(x, s)$ we see that

$$
L_{\gamma} u_{3}(a, s) \neq 0
$$

Otherwise

$$
n \geq S\left(u_{3}, a^{+}\right)+\langle n(s)\rangle+S\left(u_{3}, b^{-}\right) \geq(\gamma+3)+2 k+(n-\gamma-2 k-1)=n+2 .
$$

Also

$$
L_{2 k} u_{3}\left(s_{0}, s_{0}\right) \neq 0 \text {. }
$$

For if $L_{2 k} u_{3}\left(s_{0}, s_{0}\right)=0$, then $L_{2 k+1} u_{3}\left(s_{0}, s_{0}\right) \neq 0$. Otherwise (4) of Theorem 3 is violated. For the same reason

$$
k L_{2 k+1} u_{1}\left(s_{0}, s_{0}\right)=L_{2 k+1} u_{2}\left(s_{0}, s_{0}\right) \neq 0 .
$$

Thus, there is a linear combination of $u_{2}$ and $u_{3}$, say $z$, such that $L_{i} z\left(s_{0}, s_{0}\right)=0$ for $i=0,1, \ldots, 2 k+1$, which again violates (4) of Theorem 3 .

The Wronskian

$$
\omega\left(L_{i}\left(u_{2}\left(x, s_{0}\right)\right), L_{i}\left(u_{3}\left(x, s_{0}\right)\right)\right) \neq 0 \quad \text { for } x \in\left(a, s_{0}\right), i=0, \ldots, n-1 .
$$

Otherwise, there is a linear combination $z$ of $u_{2}$ and $u_{3}$ such that $L_{i} z$ has a double zero at $s_{1}$ with $a<s_{1}<s_{0}$. Thus by (5)

$$
\begin{aligned}
n & \geq S\left(z, \dot{a}^{+}\right)+\left\langle n\left(s_{1}\right)\right\rangle+\left\langle n\left(s_{0}\right)\right\rangle+S\left(z, b^{-}\right) \\
& \geq(\gamma+1)+2+(2 k)+(n-\gamma-2 k-1)=n+2 .
\end{aligned}
$$

We next show that if $u_{1}\left(x, s_{0}\right)$ has $t$ zeros on $\left(a, s_{0}\right)$, then $u_{3}\left(x, s_{0}\right)$ has $t+1$ zeros on $\left(a, s_{0}\right)$. By $(14)$ the zeros of $u_{3}\left(x, s_{0}\right)$ and $u_{2}\left(x, s_{0}\right)=k u_{1}\left(x, s_{0}\right)$ separate on $\left(a, s_{0}\right)$. Thus it is enough to show that if $s_{1}$ and $s_{2}$ are two consecutive zeros of $u_{1}\left(x, s_{0}\right)$ with either $s_{1}=a$ or $s_{2}=s_{0}$ on $\left[a, s_{0}\right]$, then $u_{3}\left(x, s_{0}\right)$ has a zero on $\left(s_{1}, s_{2}\right)$. Suppose $u_{3}\left(x, s_{0}\right) \neq 0$ for $x \in\left(s_{1}, s_{2}\right)$. Then $h(x) \equiv u_{1}\left(x, s_{0}\right) / u_{3}\left(x, s_{0}\right)$ is continuous on $\left(s_{1}, s_{2}\right)$. Now $u_{1}\left(x, s_{0}\right)$ has a zero of order exactly $\gamma+1$ at $a$, and by $(9)$ and $(11) u_{3}\left(x, s_{0}\right)$ has a zero of order exactly $\gamma$. At $x=s_{0}, u_{1}\left(x, s_{0}\right)$ and $u_{3}\left(x, s_{0}\right)$ have zeros of order exactly $2 k+1$ and $2 k$, respectively. Thus, defining $h\left(s_{1}\right)=h\left(s_{2}\right)=0$, we see by l'Hospital's rule that $h(x)$ is continuous on $\left[s_{1}, s_{2}\right]$. Since $h(x) \neq 0$ for $x \in\left(s_{1}, s_{2}\right), h$ must have an extreme point at $\bar{s} \in\left(s_{1}, s_{2}\right)$ at which $(h)^{\prime}(\bar{s})=0$. It follows that

$$
u_{3}\left(\bar{s}, s_{0}\right) L_{1} u_{1}\left(\bar{s}, s_{0}\right)-u_{1}\left(\bar{s}, s_{0}\right) L_{1} u_{3}\left(\bar{s}, s_{0}\right)=0 .
$$


Thus $z(x) \equiv u_{3}\left(x, s_{0}\right) h(\bar{s})-u_{1}\left(x, s_{0}\right)$ is such that $L_{0} z(\bar{s})=L_{1} z(\bar{s})=0$, which is not possible by $(14)$. Thus $u_{3}\left(x, s_{0}\right)$ has a zero in $\left(s_{1}, s_{2}\right)$.

By $(11) L_{\gamma} u_{3}\left(a, s_{0}\right) \neq 0$. Thus assume, without loss of generality, $L_{\gamma} u_{3}\left(a, s_{0}\right)>$ 0 . Since $L_{i} u_{3}\left(a, s_{0}\right)=0$ for $i=0, \ldots, \gamma-1$, it follows that $L_{i} u_{3}\left(a^{+}, s_{0}\right)>0$ for $i=0, \ldots, \gamma$. Since by (8) and (9) $L_{\gamma+1} u_{1}\left(a, s_{0}\right)=-L_{\gamma} u_{3}\left(a, s_{0}\right)$, it follows that $L_{\gamma+1} u_{1}\left(a, s_{0}\right)<0$. Since $L_{i} u_{1}\left(a, s_{0}\right)=0$ for $i=0, \ldots, \gamma$, it follows that $L_{i} u_{1}\left(a^{+}, s_{0}\right)<0$ for $i=0, \ldots, \gamma+1$. Suppose $u_{1}\left(x, s_{0}\right)$ has $t$ zeros, which by (10) and (13) are necessarily simple, in $\left(a, s_{0}\right)$. Then $(-1)^{t+1} u_{1}\left(s_{0}^{-}, s_{0}\right)>0$. Since $L_{i} u_{1}\left(s_{0}, s_{0}\right)=0$ for $i=0, \ldots, 2 k$, it follows that

$$
(-1)^{t+1+i} L_{i} u_{1}\left(s_{0}^{-}, s_{0}\right)>0 \text { for } i=0, \ldots, 2 k+1 .
$$

Since $u_{3}\left(x, s_{0}\right)$ has $t+1$ simple zeros in $\left(a, s_{0}\right)$, then $(-1)^{t+1} u_{3}\left(s_{0}^{-}, s_{0}\right)>0$. Since $L_{i} u_{3}\left(s_{0}, s_{0}\right)=0$ for $i=0,1, \ldots, 2 k-1$, it follows that

$$
(-1)^{t+1+i} L_{i} u_{3}\left(s_{0}^{-}, s_{0}\right)>0 \text { for } i=0, \ldots, 2 k .
$$

By $(7)$ and (9) $L_{\gamma+1} u_{2}\left(a, s_{0}\right)=L_{2 k} u_{3}\left(s_{0}, s_{0}\right)$. Now

$$
\frac{\partial U\left(a, s_{0}\right)}{\partial s}=\frac{L_{2 k+1} u_{1}\left(s_{0}, s_{0}\right)}{\rho_{2 k+1}\left(s_{0}\right)} \text { and } \quad \frac{\partial U\left(a, s_{0}\right)}{\partial x}=\frac{L_{\gamma+1} u_{2}\left(a, s_{0}\right)}{\rho_{\gamma+1}(a)}=\frac{L_{2 k} u_{3}\left(s_{0}, s_{0}\right)}{\rho_{\gamma+1}(a)} \text {. }
$$

Thus

$$
\frac{\partial U\left(a, s_{0}\right) / \partial s}{\partial U\left(a, s_{0}\right) / \partial x}=\frac{(-1)^{t+2 k+2} L_{2 k+1} u_{1}\left(s_{0}, s_{0}\right) / \rho_{2 k+1}\left(s_{0}\right)}{(-1)(-1)^{t+2 k+1} L_{2 k} u_{3}\left(s_{0}, s_{0}\right) / \rho_{\gamma+1}(a)}<0
$$

by $(15),(16),(12)$ and (13). Hence $d x /\left.d s\right|_{s=s_{0}}>0$.

We define

$$
D(x) \equiv\left|\begin{array}{ccc}
L_{0} y_{\gamma+1}(x) & \cdots & L_{0} y_{\gamma+2 k+1}(x) \\
\vdots & & \vdots \\
L_{2 k} y_{\gamma+1}(x) & \cdots & L_{2 k} y_{\gamma+2 k+1}(x)
\end{array}\right|
$$

THEOREM 5. There is an $s_{0} \in(a, b)$ such that $D\left(s_{0}\right)=0$ if and only if an $U\left(a, s_{0}\right)=0$.

PROOF. Since $y_{i}$ has a zero of order exactly $i$ at $x=a$, expanding $U\left(a, s_{0}\right)$ by the last row we have $U\left(a, s_{0}\right)=-L_{\gamma} y_{\gamma}(a) D\left(s_{0}\right)$.

To show a connection between the zeros of $D(x)$ and disconjugacy properties of (4), we need some information about the distribution of zeros of $L_{i} u_{2}(x, s)$.

THEOREM 6. Let $s \in(a, b)$ and $u_{2}(x, s)$ be given by $(7)$. Then

(i) there exists a first zero $x_{i}$ of $L_{i} u_{2}(x, s)$ in $(a, s)$ for $i=1, \ldots, \gamma$,

(ii) $x_{\gamma}<x_{\gamma-1}<\cdots<x_{1}$,

(iii) the simple zeros of $L_{j} u_{2}(x, s)$ are differentiable functions of $s$, and

(iv) if $D\left(s_{0}\right)=0$ for $s_{0} \in(a, b)\left(D(x)\right.$ given by (17)) and if $s>s_{0}$, then there is $x_{0} \in(a, s)$ such that $u_{2}\left(x_{0}, s\right)=0$.

PROOF. Since $L_{j} u_{2}(a, s)=0$ for $j=0, \ldots, \gamma-1$, if $L_{j} u_{2}(s, s)=0$ for $j=$ $0, \ldots, \gamma-1$, then (i) follows from Rolle's Theorem. Suppose $r<\gamma-1$ is the largest integer such that $L_{j} u_{2}(s, s)=0$ for $j=0, \ldots, r$. Then (i) holds for $i=r+1, \ldots, \gamma$ by applying Rolle's Theorem $i-r$ times. For $i=1, \ldots, r$ (i) follows from a single application of Rolle's Theorem. 
Since $L_{t} u_{2}(a, s)=0$ implies $L_{t} u_{2}(x, s)$ and $L_{t+1} u_{2}(x, s)$ have the same sign on a right neighborhood of $a, L_{i}\left(u_{2}(a, s)\right)=0$ for $i=1, \ldots, \gamma-1$, and $N\left(u_{2}(x, s)\right)=n$, (ii) follows by Theorem 2 .

Part (iii) follows directly from the definition (7) of $u_{2}(x, s)$ and the Implicit Function Theorem.

Let $x_{i}$ be the zero of $L_{i} u_{2}\left(x, s^{*}\right)$, for $s^{*}<s_{0}$, given by (i) for $i=1,2, \ldots, \gamma$. By Theorem $4, x_{\gamma}$ cannot exit $(a, s)$ through $a$ as $s$ increases from $s^{*}$. Also $x_{1}$ cannot exit $(a, s)$ through $s$; otherwise $u_{2}(x, s)$ will have a zero of order at least $2 k+2$ at $s$, which implies $N\left(u_{2}(x, s)\right)>n$. By $(14) L_{i} u_{2}(x, s)$ cannot have a multiple zero in $(a, s)$. Thus the order of the zeros given by (ii) must be maintained as $s$ increases from $s^{*}$. Consequently none of the zeros of (i) can leave $(a, s)$ as $s$ increases from $s^{*}$.

By Theorem 4 and Theorem 5 , a zero $t_{\gamma}$ of $L_{\gamma} u_{2}(x, s)$ enters $(a, s)$ through $a$ as $s$ increases through $s_{0}$. By Theorem $2, L_{t+1} u_{2}(x, s)$ has exactly one sign change between two consecutive zeros of $L_{t} u_{2}(x, s)$ for $x \in[a, b]$. Thus we conclude $L_{\gamma-1} u_{2}(x, s)$ must have a zero, $t_{\gamma-1}$, between $t_{\gamma}$ and $x_{\gamma}$. By (ii) $t_{\gamma-1} \neq x_{\gamma-1}$ repeatedly applying Theorem 2 and (ii); we conclude that $u_{2}\left(x_{0}, s\right)=0$ for some $x_{0} \in(a, s)$.

THEOREM 7. Suppose there is an $s_{0} \in(a, b)\left(s_{0} \in(a,+\infty)\right)$ such that $D\left(s_{0}\right)=$ 0 (where $D(x)$ is defined by (17)). Then (4) is not $(\gamma+1, n-\gamma-1)$ disconjugate on $[a, b]([a,+\infty])$.

PROOF. We will first prove the theorem for the finite case. Let $y(x, s)=$ $\alpha(s) u_{2}(x, s)$ be such that $\sum_{i=0}^{n-1} L_{i}^{2} y(a, s)=1$. Then by standard compactness arguments there is a sequence $\left\{s_{i}\right\}_{i=1}^{\infty}$ such that $\lim _{i \rightarrow \infty} s_{i}=b$ and $\lim _{i \rightarrow \infty} y\left(x, s_{i}\right)=$ $z(x)$ is a nontrivial solution of (4) and convergence is uniform on $[a, b]$. It follows that $z(x)$ has zeros of order at least $\gamma$ at $a$ and $n-\gamma-1$ at $b$. By Theorem 6, for each $s \in\left(s_{0}, b\right), u_{2}(x, s)$, and thus $y(x, s)$, has a zero in $(a, s)$. Letting $x(s)$ denote the zero of $y(x, s)$ in $(a, s)$, there is a subsequence of $\left\{s_{i}\right\}$, say $\left\{s_{i_{k}}\right\}$, such that $\lim _{k \rightarrow \infty} x\left(s_{i_{k}}\right)=x^{*} \in[a, b]$. Further $z\left(x^{*}\right)=0$. Now $x^{*} \neq b$; otherwise $z$ has a zero of order at least $n-\gamma$ at $b$, and (4) would have an $(\gamma, n-\gamma)$ interval of oscillation, which is impossible. If $x^{*}=a$, then $z$ has a zero of order at least $\gamma+1$ at $a$, and thus (4) is not $(\gamma+1, n-\gamma-1)$ disconjugate.

If $x^{*} \in(a, b)$, define

$$
z(x, s)=\left|\begin{array}{ccc}
L_{0} z_{1}(a) & \cdots & L_{0} z_{n}(a) \\
\vdots & & \vdots \\
L_{\gamma-1} z_{1}(a) & & L_{\gamma-1} z_{n}(a) \\
& & \\
L_{0} z_{1}(s) & & L_{0} z_{n}(s) \\
\vdots & & \vdots \\
L_{n-\gamma-2} z_{1}(s) & & L_{n-\gamma-2} z_{n}(s) \\
& & \\
z_{1}(x) & \cdots & z_{n}(x)
\end{array}\right|
$$


where $z_{1}, \ldots, z_{n}$ is a basis for the solution space of (4). Since $z$ has zeros of order $\gamma$ and $n-\gamma-1$ at $a$ and $b$, respectively, and such solutions are essentially unique, it follows that $z(x, b)=k z(x)$. Thus $z\left(x^{*}, b\right)=0$. Since $N(z(x, s))=n$, it follows that the zeros of $z(x, s)$ in $(a, s)$ are simple and thus as in Theorem 6 are differentiable functions of $s$. Let $x(s)$ be the simple zero of $z(x, s)$ so that $x(b)=x^{*}$. For $s$ close to $a, z(x, s)$ has no zeros in $(a, s)$. Now $x(s)$ cannot enter $(a, s)$ through $s$ as $s$ increases to $b$ since a $(\gamma, n-\gamma)$ interval of oscillation is impossible. Thus $x(s)$ must enter $(a, s)$ through $a$, and thus $(4)$ is not $(\gamma+1, n-\gamma-1)$ disconjugate on $[a, b]$.

For the infinite case, we consider the functions $y_{i}(x, m)$ where

$$
y_{i}(x)=\lim _{m \rightarrow \infty} y_{i}(x, m)
$$

as in Theorem 3. Letting

$$
D_{m}(x) \equiv\left|\begin{array}{ccc}
L_{0} y_{\gamma+1}(x, m) & \cdots & L_{0} y_{\gamma+2 k+1}(x, m) \\
\vdots & & \vdots \\
L_{2 k} y_{\gamma+1}(x, m) & \cdots & L_{2 k} y_{\gamma+2 k+1}(x, m)
\end{array}\right|
$$

we have

$$
\lim _{m \rightarrow \infty} D_{m}(x)=D(x) \equiv\left|\begin{array}{ccc}
L_{0} y_{\gamma+1}(x) & \cdots & L_{0} y_{\gamma+2 k+1}(x) \\
\vdots & & \vdots \\
L_{2 k} y_{\gamma+1}(x) & \cdots & L_{2 k} y_{\gamma+2 k+1}(x)
\end{array}\right|
$$

Further, since $y_{i}(x, m)$ and its quasi-derivatives converge uniformly on compact intervals, $D_{m}(x)$ converges uniformly to $D(x)$ on compact intervals. If $D\left(s_{0}\right)=0$, then $D^{\prime}\left(s_{0}\right) \neq 0$. Otherwise there is a solution $u \in \operatorname{span}\left\{y_{\gamma+1}, \ldots, y_{\gamma+2 k+1}\right\}$ with a zero of multiplicity $2 k+2$ at $s_{0}$ or $\gamma+2$ at $a$. But either alternative is impossible by Theorem 3 and Theorem 2 . Thus since $D(x)$ changes signs at $s_{0}$, there is a large $m$ such that $D_{m}(x)=0$ for some $x \in(a, m)$. Thus from the finite case (4) is not $(\gamma+1, n-\gamma-1)$ disconjugate on $[a, m]$ and hence not on $[a,+\infty)$.

THEOREM 8. If (4) is not $(\gamma+1, n-\gamma-1)$ disconjugate on $[a, b]$ and if $b$ is not $a(\gamma+1, n-\gamma-1)$ conjugate point of $a$, then $D(x)$ must have a zero in $(a, b)$.

Proof. By Elias [2, Theorem 3], it is enough to show that $D(x) \neq 0$ on $(a, b)$ implies there is a solution of (4) with zeros of multiplicities $\gamma$ and $n-\gamma-1$ at $a$ and $b$, respectively, with no zeros in $(a, b)$.

There is an $s>a$ such that $u_{2}(x, s) \neq 0$ for $x \in(a, s)$. As $s$ increases from $a$, a zero cannot enter $(a, s)$ through $s$. Otherwise for some $s_{1} \in(a, b)$, $L_{2 k+1} u_{2}\left(s_{1}, s_{1}\right)=0$ and by $(5)$

$$
\begin{aligned}
N\left(u_{2}\left(x, s_{1}\right)\right) & \geq S\left(u_{2}, a^{+}\right)+S\left(u_{2}, b^{-}\right)+\left\langle n\left(s_{1}\right)\right\rangle \\
& \geq(\gamma+1)+[n-(\gamma+2 k+1)]+2 k+2=n+2 .
\end{aligned}
$$

Thus if $u_{2}(x, s)$ has a zero in $(a, s)$, it must enter through $a$ as $s$ increases. But that means $L_{\gamma} u_{2}\left(a, s_{2}\right)=0$ for some $s_{2} \in(a, b)$. But by Theorem 5 and (7) that is possible only when $D\left(s_{2}\right)=0$. Thus for every $s \in(a, b), u_{2}(x, s) \neq 0$ for $x \in(a, s)$. Letting $y(x, s)=\alpha(s) u_{2}(x, s)$ as in the proof of Theorem 7 and letting $s$ approach $b$ along a suitable sequence, we obtain a solution $z(x)$ of $(4)$ with zeros of multiplicities at least $\gamma$ and $n-\gamma-1$ at $a$ and $b$, respectively. But since $b$ is not 
a $(\gamma+1, n-\gamma-1)$ conjugate point of $a$, the multiplicities of the zeros above must be exact. If $z\left(x_{1}\right)=0$ for $x_{1} \in(a, b)$, then $L_{1} z\left(x_{1}\right)=0$ also. But by Theorem 2 that is not possible. Thus $z(x)$ has no zeros on $(a, b)$.

An immediate corollary of Theorem 8 is

COROLlary 1. If $D(x) \neq 0$ for $x \in(a, b)$, then (4) is disconjugate on $[a, c]$ for every $c<b$.

Defining $D(x)$ by (17) using the solutions $y_{i}(x)$ for $i=0, \ldots, n-1$ of $(4)$ given by Theorem 3 with $b=+\infty$, we obtain the following version of Theorem 8 for the infinite interval.

THEOREM 9. If $D(x) \neq 0$ for $x \in(a,+\infty)$, then any solution $u$ of $(4)$ with $S\left(u, x^{+}\right)=\gamma+1$ eventually, is nonoscillatory.

PROOF. We define $u_{2}(x, s)$ by $(7)$, using the solutions $y_{i}(x)$ for $i=0, \ldots, n-1$ of (4) given by Theorem 3 with $b=+\infty$. Now $S\left(u, x^{+}\right)$is an integer valued nondecreasing function, while $S\left(u, x^{-}\right)$is nonincreasing. According to Theorem 3, $S\left(u_{2}(x, s), x^{+}\right) \geq \gamma+1$, while $S\left(u_{2}(x, s), x^{-}\right) \geq n-(\gamma+2 k+1)$ for $x \in(a,+\infty)$. Letting $x_{1} \in[a, s)$ and $x_{2} \in(s,+\infty)$, we have by (5) that

$$
\begin{aligned}
n & \geq S\left(u_{2}(x, s), x_{1}^{+}\right)+\langle n(s)\rangle+S\left(u_{2}(x, s), x_{2}^{-}\right) \\
& =(\gamma+1+j)+2 k+[n-(\gamma+2 k+1)+l] \text { for } j, l \geq 0 .
\end{aligned}
$$

Thus $n \geq n+j+l$, which implies $j=l=0$. Thus $S\left(u_{2}(x, s), x_{1}^{+}\right)=\gamma+1$, while $S\left(u_{2}(x, s), x_{2}^{-}\right)=n-(\gamma+2 k+1)$.

There is an $s>a$ such that $u_{2}(x, s) \neq 0$ for $x \in(a, s)$. Otherwise by taking the limit as $s$ approaches $a$ and normalizing as in Theorem 7, we obtain a solution $u$ of (4) with a zero of multiplicity $\gamma+2 k+2$ at $a$ with $S\left(u, x^{-}\right)=n-(\gamma+2 k+1)$ for $x>a$, which violates (5). Thus exactly as in the finite case (Theorem 8$) u_{2}(x, s) \neq 0$ for $x \in(a, s)$ and $s \in(a,+\infty)$. Taking the limit as $s$ goes to $+\infty$ and normalizing as in Theorem 7 , we obtain a solution $u$ of (4) such that $S\left(u, x^{+}\right)=\gamma+1$ for $x \in[a,+\infty)$ with no zeros in $(a,+\infty)$. According to Elias [2], this implies the desired conclusion.

4. In this section we will show that $(\gamma+2 k+1, n-\gamma-2 k-1)$ disconjugacy of (4) on $[a, b]([a,+\infty))$ implies the Wronskian of $y_{\gamma}, \ldots, y_{\gamma+2 k}$ for $0 \leq k \leq(n-\gamma-1) / 2$ has no zeros in $(a, b)((a,+\infty))$, where $y_{i}(x)$ are the solutions of $(4)$ given by Theorem 3.

The results here will parallel those of $\S 3$. Hence the proofs will be omitted.

We define functions $H$ and $T$ to play the role of $U$ and $D$ of $\S 3$ in the following way:

$$
H(x, s) \equiv\left|\begin{array}{ccc}
L_{0} y_{\gamma}(s) & \cdots & L_{0} y_{\gamma+2 k+1}(s) \\
\vdots & & \vdots \\
L_{2 k} y_{\gamma}(s) & & L_{2 k} y_{\gamma+2 k+1}(s) \\
& & \\
L_{n-\gamma-2 k-2} y_{\gamma}(x) & \cdots & L_{n-\gamma-2 k-2} y_{\gamma+2 k+1}(x)
\end{array}\right|
$$


and

$$
T(x) \equiv\left|\begin{array}{ccc}
L_{0} y_{\gamma}(x) & \cdots & L_{0} y_{\gamma+2 k}(x) \\
\vdots & & \vdots \\
L_{2 k} y_{\gamma}(x) & \cdots & L_{2 k} y_{\gamma+2 k}(x)
\end{array}\right|
$$

In Theorem 11 and below we will need the added condition that

$$
L_{n-\gamma-2 k-2} y_{\gamma+2 k}(b)=0 .
$$

If $b$ is not a $(\gamma+2 k+1, n-\gamma-2 k-1)$ conjugate point of $a$, there is no loss in generality in making that assumption. If $b$ is a $(\gamma+2 k+1, n-\gamma-2 k-1)$ conjugate point of $a$, then $L_{n-\gamma-2 k-2} y_{\gamma+2 k+1}(b)=0$, and we can interchange the role of $y_{\gamma+2 k}$ and $y_{\gamma+2 k+1}$ in the statement of the theorems of this section. Thus throughout this section we will assume $L_{n-\gamma-2 k-2} y_{\gamma+2 k}(b)=0$.

THEOREM 10. Suppose for some $s_{0} \in(a, b), H\left(b, s_{0}\right)=0$. Then the function $x(s)$ defined by $H(x, s)=0$, where $x\left(s_{0}\right)=b$, is such that $d x /\left.d s\right|_{s=s_{0}}>0$.

Using the definitions of $H$ and $T$, we have the following analog of Theorem 5 .

THEOREM 11. There is an $s_{0} \in(a, b)$ such that $T\left(s_{0}\right)=0$ if and only if $H\left(b, s_{0}\right)=0$.

THEOREM 12. Set $s \in(a, b)$ and let $u_{2}(x, s)$ be given by $(7)$. Then

(i) there is a last zero $x_{i}$ of $L_{i} u_{2}(x, s)$ in $(s, b)$ for $i=1,2, \ldots, n-\gamma-2 k-2$,

(ii) $x_{1}<x_{2}<\cdots<x_{n-\gamma-2 k-2}$,

(iii) if $T\left(s_{0}\right)=0$ for $s_{0} \in(a, b)$ and if $s<s_{0}$, then $u_{2}\left(x_{0}, s\right)=0$ for some $x_{0} \in(s, b)$.

As in $\S 3$, we have

THEOREM 13. Suppose there is an $s_{0} \in(a, b)((a,+\infty))$ such that $T\left(s_{0}\right)=0$. Then (4) is not $(\gamma+2 k+1, n-\gamma-2 k-1)$ disconjugate on $[a, b]([a,+\infty))$.

THEOREM 14. If (4) is not $(\gamma+2 k+1, n-\gamma-2 k-1)$ disconjugate on $[a, b]$ and if $b$ is not $a(\gamma+2 k+1, n-\gamma-2 k-1)$ conjugate point of $a$, then $T(x)$ must have a zero in $(a, b)$.

5. We now apply the results of $\S \S 2,3$ and 4 to give some specific factorizations of (2). We will assume the following disconjugacy conditions for (2) on $I$, where $I=[a, b]$ or $[a,+\infty)$.

(A) (2) is not $(\gamma+1+2 k, n-\gamma-1-2 k)$ disconjugate on $[a, c]$ for some $c<b$, for $k=0, \ldots, j_{0}$;

(2) is $(i, n-i)$ disconjugate on $I$ for $i<\gamma+1$ or $i>\gamma+1+2 j_{0}$.

As shown in [4] for some ray $[b,+\infty)$ and in [3] for finite intervals, our assumption is exactly what happens when $L_{n} y \equiv y^{(n)}$. For example, if $n$ is even but not divisible by $4, p(x)>0, I=[a,+\infty)$ and $y^{\prime \prime}+\left[x^{(n-2)} /(n-2) !\right] p(x) y=0$ is disconjugate, then $(2)$ is $(1, n-1)$ disconjugate on $I[5]$. However, if

$$
y^{\prime \prime}+\left[\left(\int_{t}^{\infty}(s-t) p(s) d s\right) /\left(\frac{n}{2}-2\right) !\left(\frac{n}{2}-1\right) !\right] y=0
$$


is oscillatory, then (2) is not $(n / 2, n / 2)$ disconjugate on $I[5]$. Thus by [3] there is a $\gamma$ such that $(2)$ is not $(\gamma+1+2 k, n-\gamma-1-2 k)$ disconjugate on $I$ for $k=0, \ldots, n / 2-\gamma-1$ but is $(i, n-i)$ disconjugate on $I$ for $i<\gamma+1$ or $i>n-\gamma-1$.

The factorization for (2) given in our next theorem can easily be obtained by using methods similar to Theorem 3 . However, using theorems of $\S \S 3$ and 4, we will show other factorizations are possible.

THEOREM 15. If the operator (2) satisfies assumption $(A)$, then it admits a factorization on the interior of $I$ of the form

$$
L=L_{0} \cdots L_{\gamma-1} Q_{0} \cdots Q_{j_{0}} L_{\gamma+2 j_{0}+2} \cdots L_{n-1}
$$

where the $L_{i}$ are first and the $Q_{i}$ are second order operators of the form (1) with each $Q_{i}$ irreducible.

PROOF. Let $y_{0}, y_{1}, \ldots, y_{n-1}$ be as in Theorem 3 . Then

$$
W\left(y_{i}, y_{i+1}, \ldots, y_{n-1}\right)\left(x_{0}\right)=0
$$

for $x_{0}>a$ if and only if there is a $z$ in $\operatorname{span}\left\{y_{i}, y_{i+1}, \ldots, y_{n-1}\right\}$ with a zero of multiplicity $n-i$ at $x_{0}$. But since $z$ has a zero of multiplicity $i$ at $a$, it follows that $W\left(y_{i}, y_{i+1}, \ldots, y_{n-1}\right)\left(x_{0}\right)=0$ for $x_{0}>a$ if and only if (2) is not $(i, n-1)$ disconjugate on $I$. Thus repeatedly using Theorem 1 , it follows that (2) admits factorization (21). To see that $Q_{l}$ is irreducible, we observe that $z_{1} \equiv W\left(y_{\gamma+2 l+1}, y_{\gamma+2 l+2}, \ldots, y_{n-1}\right)$ and $z_{2} \equiv W\left(y_{\gamma+2 l}, y_{\gamma+2 l+2}, \ldots, y_{n-1}\right)$ are independent solutions of $Q_{l} y=0$. Since $(2)$ is not $(2 l+1+\gamma, n-2 l-1-\gamma)$ disconjugate on $I$, it follows by the first part of the proof that $z_{1}$ must have a zero at some $x_{1}>a$. But since the order of the zero of any other solution $z$ of $Q_{l} y=0$ is less than that of $z_{1}$ at $a, z$ must have a zero at some $x_{2}$ where $a<x_{2}<x_{1}$. Thus $Q_{l}$ fails to be disconjugate and hence by the classical result of Pólya [5], cannot be factored.

Although (21) seems to be a natural way to factor (2), our next theorem shows that it is not the only way.

THEOREM 16. If the operator (2) satisfies assumption $(A)$, then it admits a factorization on the interior of $I$ of the form

$$
L=L_{0} \cdots L_{\gamma-1} L_{\gamma+2 j_{0}+2} \cdots L_{n-1} Q_{0} \cdots Q_{j_{0}}
$$

where the $L_{i}$ are first and the $Q_{i}$ second order operators of the form (1) with each $Q_{i}$ irreducible.

PROOF. Let $y_{0}, y_{1}, \ldots, y_{n-1}$ be a basis for the solution space of $L y=0$ as in Theorem 3. Then as in the first part of Theorem $15, W\left(y_{i}, y_{i+1}, \ldots, y_{n-1}\right)(x) \neq 0$ for $x \in \operatorname{int} I$ and for $i=1, \ldots, \gamma$ since $(2)$ is $(i, n-i)$ disconjugate on $I$. Thus applying Theorem 1 repeatedly, $L=L_{0} \cdots L_{\gamma-1} Q$, where $y_{\gamma}, y_{\gamma+1}, \ldots, y_{n-1}$ is a fundamental set of solutions for $Q y=0$. Now applying Theorem 3 for even order Wronskians and Theorem 13 for odd order Wronskians, $W\left(y_{\gamma}, y_{\gamma+1}, \ldots, y_{j}\right)(x) \neq 0$ for $x \in \operatorname{int} I$ for $x \in \operatorname{int} I$ since $(2)$ is $(\gamma+j, n-\gamma-j)$ disconjugate for $j \geq$ $\gamma+1+2 j_{0}$. Again applying Theorem 1 repeatedly, $Q=L_{\gamma+2 j_{0}+2} \cdots L_{n-1} P$, where $y_{\gamma}, y_{\gamma+1}, \ldots, y_{\gamma+2 j_{0}+l}$ is a fundamental set of solutions for $P y=0$. Applying Theorem 3 , we see

$$
W\left(y_{\gamma+2 r}, y_{\gamma+2 r+1}, \ldots, y_{\gamma+2 j_{0}+1}\right)(x) \neq 0
$$


for $x \in \operatorname{int} I$ and $r=1, \ldots, j_{0}$. Thus again using Theorem $1, P=Q_{0} \cdots Q_{j_{0}}$, where each $Q_{i}$ is second order. To see that $Q_{l}$ is irreducible, we observe that

$$
z_{1}=W\left(y_{\gamma_{+} 2 l+1}, y_{\gamma+2 l+2}, \ldots, y_{\gamma+2 j_{0}+1}\right)
$$

and

$$
z_{2}=W\left(y_{\gamma+2 l}, y_{\gamma+2 l+2}, y_{\gamma+2 l+3}, \ldots, y_{\gamma+2 j_{0}+1}\right)
$$

are independent solutions of $Q_{l} y=0$. Since $(2)$ is not $(2 l+1+\gamma, n-2 l-1-\gamma)$ disconjugate, by the corollary to Theorem $8, z_{1}$ must have a zero at $x_{1} \in$ int $I$. But since the order of the zero of any other solution $z$ of $Q_{l} y=0$ is less than that of $z_{1}$ at $a, z$ must have a zero at some $x_{2}$ where $a<x_{2}<x_{1}$. Thus $Q_{l}$ fails to be disconjugate and hence cannot be factored.

COROLLARY. If the operator (2) satisfies assumption $(A)$, then it admits a factorization on the interior of $I$ of the form

$$
L=P Q,
$$

where $P$ is disconjugate and every solution of $Q y=0$ oscillates.

Proof. Let $Q=Q_{0} \cdots Q_{j_{0}}$. Then $y_{\gamma}, y_{\gamma+1}, \ldots, y_{\gamma+2 j+1}$ is a fundamental set of solutions for $Q y=0$. Thus by Theorem 3 every solution of $Q y=0$ oscillates. Letting $P=L_{0} \cdots L_{\gamma-1} L_{\gamma+2 j+2} \cdots L_{n-1}$, it follows that $P$ is disconjugate.

Applying the results of $\S \S 3$ and 4 , it is clear that many other factorizations are possible.

EXAMPLE. Let $L y \equiv y^{(4)}-y, L_{1} y \equiv-y^{\prime} / 2 e^{x}, L_{2} y \equiv e^{x}\left[y^{\prime}-y\right]$ and $Q_{1} y=$ $-2 y^{\prime \prime}+2 y^{\prime}-4 y$. Then $L y=L_{1} \circ Q_{1} \circ L_{2}(y)$ as in Theorem 15. But if $L_{3} y \equiv$ $2 e^{x} y^{\prime}-2 e^{x} y$ and $Q_{2} y=-y^{\prime \prime}-y$, then $L y=L_{1} \circ Q_{2} \circ L_{3} y$ as in Theorem 16 .

\section{REFERENCES}

1. W. A. Coppell, Disconjugacy, Lecture Notes in Math., vol. 220, Springer-Verlag, Berlin and New York, 1971.

2. U. Elias, Oscillatory solutions and extremal points for a linear differential equation, Arch. Rational Mech. Anal. 71 (1979), 177-198. MR 81c:34027.

3. Comparison Theorems for disfocality and disconjugacy of differential equations, SIAM J. Math. Anal. 15 (1984), 922-931.

4. G. Jones, An ordering of oscillation types for $y^{(n)}+p y=0$, SIAM J. Math. Anal. 12 (1981), 72-77. MR 82a:34036.

5. ___ Oscillation properties of $y^{n}+p y=0$, Proc. Amer. Math. Soc. 78 (1980), 230-244.

6. G. Pólya, On the mean-value theorem corresponding to a given linear homogeneous differential equation, Trans. Amer. Math. Soc. 24 (1922), 312-324.

7. E. Heinz, Halbbeschränktheit gewöhnlicher Differentialoperatoren höherer Ordnung, Math. Ann. 135 (1958), 1-49. MR 21 \#743.

8. A. Zettl, Factorization of differential operators, Proc. Amer. Math. Soc. 27 (1971), 425426. MR 42 \#7966.

9. __ General theory of the factorization of ordinary linear differential operators, Trans. Amer. Math. Soc. 197 (1974), 341-353. MR 51 \#978.

Department of Mathematics, University of Houston, UNIVERSity PARK, HOUSTON, TEXAS 77004

Department of Mathematics, Murray State University, Murray, Kentucky 42071

Department of Mathematics, Texas Southern University, houston, Texas 77004 